Methods 2456 adults were surveyed in Hyderabad, Bangalore and Chandigarh in India. Socio-demographic and lifestyle characteristics were obtained through a questionnaire, and a dried blood spot (DBS) was collected from all individuals aged 18 and over; sexual behaviour was collected from those aged $18 \mathrm{e} 49$ years. DBS samples were tested for HSV-2 and syphilis serology. The association between HSV-2 and syphilis infections with socio-demographic and behavioural variables was analysed using multivariable logistic regression.

Results The prevalence of HSV-2 and syphilis was $10.1 \%$ and $1.7 \%$, respectively. Urban/rural differences in prevalence were only seen for syphilis. For both infections, the prevalence between males and females was not significantly different. In males and females, HSV-2 prevalence increased significantly with increasing age; for syphilis, a slight trend was seen only in females. In a multivariable analysis, HSV-2 infection in males and females was associated with site, religion and testing positive for syphilis, in addition to reporting \$2 lifetime partners in the previous year among males and being ever married or having had sex with a non-regular partner in the last year among females.

Conclusions The burden and geographic heterogeneity of HSV-2 and syphilis infections in India are significant. A national household and DBS-based sexually transmitted infection (STI) surveillance system would enable monitoring, especially in relation to the HIV epidemic, and planning of evidence-based prevention and treatment programmes.

\section{P1-83 PHYSICAL ACTIVITY AND THE POTENTIAL INDEPENDENT DETRIMENTAL MENTAL HEALTH OUTCOMES OF SEDENTARY BEHAVIOUR IN THE GENERAL POPULATION}

doi:10.1136/jech.2011.142976c.76

Johan Ven Der Hayden,* ${ }^{2} \mathrm{M}$ Asztalos, II De Bourdeaudhuij, ' G Cardon. 'Ghent University, Ghent, Belgium; ${ }^{2}$ Scientific Institute of Public Health, Brussels, Belgium

Introduction Sedentary behaviour (SB) is a distinctive form of human behaviour that should not be considered the endpoint of the physical activity (PA) continuum. Hence, SB and PA might work independently in relation with mental health $(\mathrm{MH})$. Investigating these relationships may inform public health initiatives targeting $\mathrm{MH}$ promotion.

Methods 6720 adults aged 24-65 years from the Belgian National Health Survey provided data on SB and PA via the IPAQ, and on $\mathrm{MH}$ via the GHQ12 and the SCL-90-R. Multiple logistic regression analyses examined associations between $\mathrm{SB}$ and five $\mathrm{MH}$ problems, controlling for PA and other confounders. Further analyses explored variations across gender, age, SES, and participation in recommended moderate- and vigorous-intensity PA.

Results In the total sample, SB was positively associated with feelings of depression $\mathrm{OR}=1.406,95 \% \mathrm{CI}$ [1.157 to 1.709 ], anxiety $\mathrm{OR}=1.523,95 \% \mathrm{CI}$ [1.217 to 1.905$]$, and symptoms of somatisation $\mathrm{OR}=1.401,95 \% \mathrm{CI}$ [1.134 to 1.732 ]. These $\mathrm{MH}$ problems were significantly more present among individuals who sat over $2100 \mathrm{~min} /$ week (controlled for PA), indicating potential independent detrimental $\mathrm{MH}$ outcomes of SB. Further, these positive associations existed independent from gender and age, in the lower SES category, and among all individuals who did not fulfil one PA recommendation. Fulfilment of either one PA recommendation, and high SES seemed protective, with the potential $\mathrm{MH}$ protective effects of recommended $\mathrm{PA}$ approaching the $\mathrm{MH}$ protective effects attributed to high SES.

Conclusion While it is important to encourage both increases in PA and reductions in $\mathrm{SB}$, from the perspective of $\mathrm{MH}$, increasing $\mathrm{PA}$ may represent the priority, since $\mathrm{PA}$ seems protective against the independent detrimental $\mathrm{MH}$ outcomes of $\mathrm{SB}$.

\section{P1-84 HIDDEN MARKOV RANDOM FIELD FOR SPATIAL AND SPATIAL-TEMPORAL RISK MAPPING}

doi:10.1136/jech.2011.142976c.77

${ }^{1,2}{ }^{2}$ Azizi, ${ }^{2} \mathrm{~F}$ Forbes, ${ }^{1} \mathrm{M}$ Charras-Garrido, ${ }^{1} \mathrm{D}$ Abrial. ${ }^{1}$ INRA, Clermont-Ferrand, France, ${ }^{2}$ INRIA, Grenoble, France

Risk mapping in epidemiology enables the epidemiologist to identify regions with high or low risk of contamination and understand the underlying mechanisms of the spread of the disease. In this work we are presenting a method of risk mapping based on finite mixture models, in which the allocation to the mixture components is modelled through a correlated process, the Potts model. The inference is performed using an approximation of the Expectation Maximisation (EM) algorithm based on the mean-field theory. One advantage of this model is that the classification of the risk is done automatically and not performed in a second step as in current risk mapping.

Methods We are presenting also a way of initialisation able to overcome the sensitivity of this algorithm to its initial parameters. Combining the proposed model to this way of initialisation is leading to good results even in the case of animal non contagious diseases, in which the risk level is very small. This is illustrated in both simulated data and real data: The bovine spongiform Encephalopathy disease in France. We will also introduce an extension of this model to the spatial-temporal context since taking in account the temporal dependencies besides the spatial ones usually provides more useful cues. This methodology will be illustrated on simulated data.

\section{P1-85 EPIDEMIOLOGICAL RISK ASSESSMENT OF C DIFFICILE OUTBREAKS LEADS TO RAPID IMPLEMENTATION OF A NATIONAL LABORATORY BASED SURVEILLANCE SYSTEM AND CHANGES IN HOSPITALS' HYGIENIC GUIDELINES}

doi:10.1136/jech.2011.142976c.78

${ }^{1} \mathrm{~S}$ Bacci, ${ }^{*}{ }^{2} \mathrm{~K}$ E P Olsen, ${ }^{1} \mathrm{~K}$ Mølbak. ${ }^{1}$ Department of Epidemiology, Statens Serum Institut, Copenhagen, Denmark; ${ }^{2}$ National Reference Laboratory for Enteropathogenic Bacteria, Department of Microbiological Surveillance and Research, Statens Serum Institute, Copenhagen, Denmark

Introduction In January 2009 the Capital region of Denmark experienced for the first time an outbreak of hypervirulent Clostridium difficile PCR ribotype 027.

Methods An epidemiological investigation was launched in a regional hospital in February 2009; the Statens Serum Institut supported the hospital in the outbreak investigation and risk assessment. Considerable media attention raised awareness among other hospitals of the Region and prompted informal collaboration. Risk management was conducted by the National Board of Health, which issued guidelines to enhance surveillance of $C$ difficile and to implement control measures.

Results In April 2009, the National Board of Health requested all Clinical Microbiology Departments to submit isolates of $C$ difficile to the Reference Laboratory of Statens Serum Institut, if those fulfilled a set of criteria. The criteria ensured surveillance of severe $C$ difficile infections by strain characterisation. An epidemiological study on mortality confirmed that the criteria used in the surveillance programme ensured detection of emerging and hyper virulent strains. Following discussion with Hospital Hygienic Committees, in collaboration with the Statens Serum Institut, the National Board of Health, and the Danish Working Environment Authority the recommendations of the Danish Working Environment Authority were changed and disinfection with chlorine became legal and was included as an option in the hospital hygienic guidelines of the Capital Region. Conclusion The outbreak response including field epidemiology investigation led to rapid changes in national surveillance and policies. Involvement of different stakeholders and communication 
between regional and national level was the key to have these interventions happen rapidly.

\section{P1-86 EPIDEMIOLOGY OF CHILDHOOD ROAD TRAFFIC INJURY: IN BANGLADESH YIELD OF THE LARGEST COMMUNITY BASED SURVEY}

doi:10.1136/jech.2011.142976c.79

\author{
${ }^{1,2}$ M D K ul Baset, ${ }^{*}$ A K M F Rahman, ${ }^{1}$ A Rahman, ${ }^{1}$ S M S R Mashreky, ${ }^{2} E$ Towner. \\ ${ }^{1}$ Centre for Injury Prevention and Research Bangladesh (CIPRB), Dhaka, Bangladesh; \\ ${ }^{2}$ University of the West of England, Bristol, UK
}

Introduction Road traffic injuries (RTIs) are a leading cause of morbidity, disability and mortality in low income countries. $93 \%$ of child road deaths occur in low and middle income countries. Good data are needed to raise awareness of the scale of the problem and to develop and target injury prevention programmes.

Objective Investigate the magnitude of and risk factors for childhood RTIs in Bangladesh.

Methodology A cross sectional study was conducted to determine the current childhood road traffic injury situation in Bangladesh. Face-to-face interviews were used. Multi stage cluster sampling was used to select the sample.

Result Nationally representative data were collected from 171366 rural and urban households. In the sampled households 351651 children aged $0-17$ years were identified; 178285 were males and 173366 females. The rate of non-fatal RTI among children under 18 years of age was calculated as 186.55 per 100000 child-year. The highest incidence (216.06/100 000 child-year) was found among the 5-9 years age group. Among the total children with non-fatal RTI $75.3 \%$ were male and $24.7 \%$ were female. The incidence of childhood RTI was found to be three times higher in rural children than urban children. Most of the childhood RTIs were pedestrian injuries. The rate of fatal RTI was 5.97 per 100000 per year among all children. Conclusion The study has confirmed that childhood RTI is a major public health problem in Bangladesh particularly in rural areas. An appropriate prevention programme is urgently required to prevent fatal and nonfatal RTIs in rural areas.

\section{P1-87 SURVEILLANCE OF DEATH FOR WOMEN OF CHILDBEARING AGE: EXPERIENCE REPORT}

doi:10.1136/jech.2011.142976c.80

M Bastos, * J Armond, S Prado. Universidade de Santo Amaro, São Paulo, SP, Brazil

Introduction Since the establishment of policies of humanisation of delivery and to obtain more accurate data on causes of maternal deaths, maternal mortality in Brazil declined. Pregnant women have been given priority in health services. As a result, we started to notice a large number of deaths of women of childbearing age, not pregnant, who now have more difficulty getting care in health services.

Objectives To identify the causes of death in women of childbearing age (10 to 49 years), to prevent them in the Public Health Unit.

Methods Time series from 2005 to 2009 that evaluated the death certificates of women of childbearing age in the region studied. After checking the main causes of death was held discussion with staff of the Unit on shares, which were immediately put into practice (task force for preventive gynaecological examinations, diagnosis of sexually transmitted diseases, and chronic degenerative diseases).

Results There were 386 deaths of women of childbearing age in the period studied. As causes, undetermined and without care (19.2\%), heart disease $10.9 \%, 10.7 \%$ violent death, cerebrovascular events $6.7 \%$, lung diseases (8.2\%), AIDS $3.6 \%$, maternal causes accounted for $2.07 \%$ and $1.55 \%$ neoplasms. The rate of death between 30 and 49 years rose from $79 \%$ in 2005 to $81 \%$ in 2009 .

Conclusions The most common causes of death were undetermined causes, cardiovascular events and violent death. It appears that prevention and health promotion carried out in health units could prevent such events.

\section{P1-88 DECREASE OF RAILWAY SUICIDES BETWEEN 1998 AND 2006: EVALUATION OF THE GERMAN RAILWAY SUICIDE PREVENTION PROJECT}

doi:10.1136/Jech.2011.142976c.81

${ }^{1} \mathrm{~J}$ Baumert, ${ }^{*} \mathrm{~K}$ Lukaschek, ${ }^{1} \mathrm{~S}$ Kunrath, ${ }^{1,2} \mathrm{~N}$ Erazo, ${ }^{1,2} \mathrm{~K} \mathrm{H}$ Ladwig. ${ }^{1}$ nstitute of Epidemiology II, Helmholtz Zentrum München, Neuherberg, Germany; ${ }^{2}$ Department of Psychosomatic Medicine and Psychotherapy, Klinikum rechts der Isar, Technische Universität München, Munich, Germany

Introduction Railway suicides are a suicidal behaviour which strongly impact psychological and socioeconomic aspects of the railway company, its employees and possible eye witnesses. The German Railway Suicide Prevention Project was carried out in 2002 aimed to prevent suicidal acts on German Railway net by a variety of measurements. The present study evaluated the impact of this project on the number of suicides during an observation period from 1998 to 2006. Methods The data base of the present study is derived from the Event Database Safety (EDS), which is the national central registry of all person accidents in the context of the national German railway company covering the entire German railway track system excluding municipal subway providers. We compared the railway suicide rate 4 years before and after starting the project by defining an "index group" (1998-2001) and a "control group" (2003-2006) using Poisson regression with estimating the average percentage change (APC).

Results The absolute number of suicidal events on the railway track system decreased from 1006 in 1998 to 724 in 2006. The mean suicide rate in the control years was $13.9 \%$ (95\% CI 6.9 to 20.4 ) lower compared to the index years $(p<0.001)$. Adjusting for the overall suicide rate attenuated the decline of the railway suicide rate (APC $4.8 \%, 95 \%$ CI 1.8 to 7.8 ) but significance remained ( $p=0.002$ ). Conclusion The present study revealed a favourable trend with decreasing railway suicide rates even taking the overall suicide rate into account. The preventive measurements carried out by the project might contribute to this development.

\section{P1-89 MILITARY POPULATIONS, MILITARY DISEASES: THE DEVELOPMENT OF MILITARY EPIDEMIOLOGY}

doi:10.1136/jech.2011.142976c.82

${ }^{1}$ B Bergman.* ${ }^{1}$ Army Medical Directorate, Camberley, UK; ${ }^{2}$ Scottish Government, Edinburgh, UK

Introduction In the mid $19^{\text {th }}$ century, military health protection began to be informed by epidemiology. This paper examines the development of military epidemiology and its impact on military health policy.

Methods Historical material drawn from military health reports and other sources is used to illustrate long-term trends and developments.

Results The science of military epidemiology can be traced back to the Scottish Enlightenment of the 18th century. At first qualitative and descriptive, the early nineteenth century saw the development of a more quantitative and analytical approach which became a powerful tool in influencing military policy to protect and improve the poor health of the Victorian soldier, who faced disease and environmental hazards far from home. Formal annual reports on the health of the Army, which were instituted in 1859 and continue to this day, have provided a unique picture of long-term health trends in a changing population. Over time, both the nature of the disease threat and the means of health protection changed, and examples will be given of trends in disease and their impact on military operational effectiveness. The military population itself also 